\title{
Causas de los accidentes de trabajo en la ciudad de Cartagena durante el período 2009 - $2012^{1}$
}

\section{Causes of accidents in the workplace in Cartagena city from 2009 to 2012}

DOI: http://dx.doi.org/10.17981/econcuc.39.1.2018.01

Artículo de investigación. Fecha de recepción: 05/12/2017-. Fecha de aceptación: 03/05/2018

\author{
Martha Yánez Contreras \\ Universidad de Cartagena \\ myanezc@unicartagena.edu.co
}

Karina Acevedo González

Universidad de Cartagena (Colombia).

kacevedog@unicartagena.edu.co

Para citar este artículo:

M. Contreras y K. Acevedo. "Causas de los accidentes de trabajo en la ciudad de Cartagena durante el período 2009 - 2012" Económicas CUC, vol. 39, no. 1, pp. 9-26, 2018 DOI: http://dx.doi.org/10.17981/econcuc.39.1.2018.01

\section{Resumen}

El objetivo de esta investigación es analizar las causas de los accidentes de trabajo en Cartagena. Tiene un enfoque cuantitativo que se implementa mediante la estimación de un modelo panel cuya unidad de análisis son los trabajadores por rama de actividad económica durante el periodo 2009 - 2012 con base en la información de la Federación de Aseguradores Colombianos y la Gran Encuesta Integrada de Hogares. Se concluye que las características propias de trabajo, como la organización de la empresa y la estabilidad laboral, inciden en la ocurrencia de los accidentes. Esto contribuye como insumo científico a investigaciones de la temática en Cartagena y Colombia.

Palabras claves: accidentes de trabajo, condiciones de trabajo, lesiones laborales, riesgos laborales, índice de accidentabilidad

\section{Abstract}

The objective this research work is to analyze causes of accidents in the workplace in the city of Cartagena, researchers of this work used quantitative methods these were implemented through assessments of patterns whose sample of procedures were workers categorized by economic activities from 2009 to 2012 , the database was provided by the Colombian Federation of Assurance along with the Great Integrated Survey of homes. As conclusion it can be said that not only particular characteristics but also the organization of companies and the laboral stability have influenced in the mentioned accidents. Further research in this area may include scientific background to study the causes of accidents on the workplace as well in the city of Cartagena as throughout Colombia.

Keywords: Accidents in the work place, work conditions, work injuries, labour risks, accident rates

\footnotetext{
${ }^{1}$ Este artículo hace parte del proyecto "Análisis de los costos y las causas de los accidentes de trabajo y su impacto en la productividad laboral en la ciudad de Cartagena para el año 2010", financiado por Colciencias y la Universidad de Cartagena para el desarrollo del programa de Jóvenes Investigadores e Innovadores "Virginia Gutiérrez de Pineda".
} 


\section{Introducción}

La economía es un sistema dinámico en el que sus tres principales agentes: la empresa, la familia y el Estado, son interdependientes. De esta forma, las empresas generan los bienes y servicios necesarios para satisfacer las necesidades de las familias. Las familias, por su parte, compran bienes y servicios para satisfacer sus necesidades. Con los ingresos percibidos por las empresas, pueden seguir con el proceso productivo, y estos, a su vez, son obtenidos por la posesión de los factores productivos. Finalmente, el Estado está facultado "para racionalizar la producción, distribución y consumo de riquezas, así como para ofrecer al trabajador colombiano la protección adecuada, de acuerdo con sus derechos adquiridos", como lo menciona el Departamento Nacional de Planeación (DNP) (1936, párr. 3). En ese sentido, el sistema económico es tan complejo que, si se presentan problemas con uno de los agentes, se obstaculiza el crecimiento y desarrollo económico del país.

Dentro de los factores que pueden provocar deficiencias en el sistema se encuentran los accidentes de trabajo. Un problema propio del mercado laboral que afecta a las empresas y a la sociedad en general por el ausentismo laboral, la pérdida de productividad y los altos costos por prestaciones económicas y sanitarias. No obstante, sus repercusiones trascienden el ámbito económico dado que afecta la vida individual y familiar de las personas involucradas. Como plantean Gomero, Llapyesan, Calizaya y Silvera (2005), el nivel de salud y seguridad en el trabajo, el desarrollo socioeconómico nacional, la calidad de vida y el bienestar de los trabajadores están estrechamente relacionados.

Según cifras de la Organización Internacional del Trabajo (OIT) (2012), más de 2,3 millones de personas en el mundo mueren cada año como resultado de una lesión mortal o enfermedades laborales, y ocurren cerca de 317 millones de accidentes laborales al año. Además, el coste total anual de estos accidentes equivale aproximadamente al $4 \%$ del
Producto Interno Bruto Mundial (OIT, 2012); situación que afecta principalmente a los países emergentes, los cuales necesitan mantener una mayor estabilidad económica para elevar su desarrollo económico (OIT, 2007).

En Colombia, durante el año 2015, ocurrieron cerca de 724 mil accidentes de trabajo, lo que significa que, de cada 100 trabajadores afiliados al régimen de seguridad social en riesgos profesionales, cerca de 7,5 presentó algún accidente de trabajo. Cabe resaltar que, durante los años 2013-2014, se presentó un crecimiento en la tasa de accidentabilidad laboral a un 10,7\%, mientras que, para el año 2015, disminuyó cinco puntos porcentuales aproximadamente, ubicándose en un 5,1\% con respecto al año 2014 (Federación de Aseguradoras Colombianas, 2018). En Cartagena, por su parte, si bien se observó una reducción en el índice de accidentalidad durante el año 2012, al situarse en 6,8 , la evolución histórica la presenta como una de las ciudades con mayores índices de accidentalidad en el país.

Según la Comunidad Andina-CAN (2003), un accidente de trabajo es todo suceso repentino que sobrevenga por causa o con ocasión del trabajo, y que produzca en el trabajador una lesión orgánica, una perturbación funcional, una invalidez o la muerte. No obstante, los tratadistas sobre el tema plantean que, si bien los accidentes laborales son repentinos, estos se pueden prevenir. Para esto, es necesaria la implementación de normas de seguridad y salud ocupacional en la empresa. Además, tal como lo sugiere Cortez (2003), aun cuando los accidentes pueden ser aleatorios, es posible identificar variables que aumentan o disminuyen la probabilidad de ocurrencia de estos.

Para el estudio de las causas de accidentalidad se han formulado diversos modelos y teorías que intentan contribuir a la generación de estrategias para su prevención. Desde el punto de vista empírico, la literatura enfatiza en dos principales grupos de causas: las que son propias de las condiciones de trabajo, también conocidas como causas micro, y las que se originan de las relaciones de trabajo, 
conocidas como causas macro (Benavides, Delclos, Benach y Serra, 2006). Estas últimas causas tienen un peso cada vez mayor en la ocurrencia de los accidentes de trabajo debido al acelerado cambio de las formas de organización del trabajo que tienden hacia una mayor flexibilidad y desregulación en el mercado laboral. También son importantes algunas características personales de los trabajadores que los hacen más propensos a la accidentalidad.

El estudio de las causas de la accidentalidad laboral en Colombia es un tema en construcción ya que las bases de datos de las administradoras de riesgos profesionales no son homogéneas; asimismo, estas bases de datos sólo contienen información sobre la población cubierta, la cual es sensible a los cambios en el empleo, y no sobre la población expuesta. De esta forma, la falta de información sobre el tiempo de trabajo de la población expuesta hace que en Colombia el indicador de accidentalidad más utilizado sea la tasa de incidencia ${ }^{2} \mathrm{y}$ tasa de accidentalidad ${ }^{3}$, en lugar del índice de frecuencia ${ }^{4} \mathrm{y}$ el índice de grave$\mathrm{dad}^{5}$. Entre otras limitaciones, se encuentra que en Colombia la unidad de análisis es la empresa, lo que impide el "conocimiento del perfil epidemiológico de la población en cobertura y distribución de afiliados por exposición a factores de riesgos" (Gallego y Correa, 2000, p. 84).

Por otro lado, la presente investigación sirve como base para la continuación de estudios en materia de accidentabilidad laboral en Colombia, y particularmente, en Cartagena, dada la escasa evidencia empírica que se encuentra en la literatura sobre dicha ciudad, sirviendo de insumo para las empresas contratantes, aseguradoras y comunidad académica.

En este orden de ideas, este artículo presenta los resultados de un análisis sobre las

\footnotetext{
${ }^{2} \mathrm{~N}^{\circ}$ accidentes $/ \mathrm{N}^{\circ}$ de trabajadores cubiertos

${ }^{3} \mathrm{~N}^{\circ}$ accidentes $/ \mathrm{N}^{\circ}$ de trabajadores

${ }^{4} \mathrm{~N}^{\circ}$ accidentes / $\mathrm{N}^{\circ}$ de horas trabajadas

${ }^{5} \mathrm{~N}^{\circ}$ de días perdidos / $\mathrm{N}^{\circ}$ de horas trabajadas
}

causas de los accidentes de trabajo en la ciudad de Cartagena teniendo en cuenta los diferentes grupos de causas que sugiere la literatura. Para ello se realiza una estimación de un modelo panel durante el periodo 2009-2012 cuya unidad de análisis son las ramas de actividad, información tomada de las estadísticas de accidentalidad de la Federación de Aseguradores Colombianos (Fasecolda). Este artículo se divide en cuatro secciones incluida esta introducción; en la segunda, se presenta la metodología; siguen los resultados, donde se pone en evidencia un análisis de la evolución y magnitud del fenómeno en Cartagena y los resultados econométricos; y, finalmente, las conclusiones.

Fundamentos teóricos y empíricos de las causas de la accidentalidad laboral

La accidentalidad laboral es un problema con importantes repercusiones en el trabajador, en su familia, en la empresa en la cual laboral y en la sociedad en general. Desde la década de los treinta, un creciente grupo de estudios buscan, desde una manera teórica y empírica, profundizar en sus desencadenantes con el objetivo de avanzar en la prevención.

El análisis teórico de las causas de accidentalidad se ha desarrollado con el surgimiento de modelos que intentan explicar de una forma cada vez más elaborada el fenómeno, aunque, hasta ahora, ninguno cuente con una aceptación unánime. Así, se pasan de modelos que dividen los comportamientos en seguros o inseguros a entender la accidentalidad como un sistema dinámico en la que confluyen personas, tareas, equipos y entorno (Saari, 1998).

Desde esta perspectiva, sobresale el modelo precursor de Heinrich (1931), el cual considera que los accidentes tienen sus causas principalmente en fallas humanas ${ }^{6}$ y que los accidentes son resultado de la ocurrencia de cinco factores en forma secuencial,

\footnotetext{
${ }^{6}$ Las condiciones peligrosas y los hechos fortuitos también son causas consideradas por Heinrich, pero éstas explican menos del $12 \%$ de los accidentes.
} 
en su orden: antecedente y entorno social; acto inseguro; falla humana; accidente; $y$, por último, daño o lesión. De este modo, así como retirar una ficha de dominó en una fila interrumpe la caída, la eliminación de uno de estos desencadenantes evita la ocurrencia de un accidente.

Este modelo ha sido el punto de partida para el desarrollo de teorías posteriores, incluso de aquellas que, en la década de los setenta, intentaron invalidar el estudio científico de las causas de la accidentalidad para incluirla en el estudio del error humano y fallas humanas. Dentro de las teorías derivadas del modelo de Heinrich (1931), se encuentra la causalidad múltiple, la cual afirma que el accidente no siempre se produce por una única causa o acción y estas causas se agrupan en dos categorías: de comportamiento (relativos al trabajador) y ambientales.

Por otra parte, la teoría de la casualidad pura, también derivada del modelo de Heinrich (1931), considera que todos los accidentes obedecen a hechos fortuitos y que todos los trabajadores de un conjunto determinado tienen la misma probabilidad de sufrir un accidente. No obstante, para la teoría de propensión al accidente existe un subconjunto de trabajadores de un conjunto general que corren un mayor riesgo. Para la teoría de probabilidad sesgada, la probabilidad de un trabajador de accidentarse en el futuro se modifica cuando se accidenta (Raouf, 1998).

El modelo de causalidad de pérdidas, desarrollado a partir del modelo de Heinrich (1931), concibe la accidentalidad como una cadena de factores o grupos de factores que producen otros de nivel inferior hasta llegar a las causas inmediatas, entre estos factores se encuentran: las condiciones materiales y medio ambiente de trabajo; deficiencias en la organización y gestión; y factores humanos. Asimismo, este modelo diferencia entre causas básicas, que son las razones reales por las cuales ocurrieron los actos y condiciones inseguras (por ejemplo, la falta de protección), y las causas inmediatas, que son aquellas que preceden inmediatamente al contacto (por ejemplo, prenda de protección incomoda)
(Botta, 2010). Otra teoría que considera que la accidentalidad se produce en cadena es el modelo causal psicosocial, el cual sugiere que la cadena de efectos recorre desde el nivel organizacional hasta el comportamiento individual de cada trabajador (Meliá, 2004).

Finalmente, otra teoría ampliamente referenciada es la teoría de homeostasis, la cual afirma que cada trabajador tiene un nivel de riesgo que mantiene constante, de manera que incrementos en la seguridad se traducen en incrementos de actos inseguros, y por tanto, los accidentes son causas y consecuencias de los cambios en el comportamiento de los operarios. Al respecto, Wilde (1998) expone que, por ejemplo, a la invención de un cigarrillo que reduce en un 50\% la probabilidad de muerte, los fumadores reaccionaran consumiendo el doble.

Por otro lado, existen los modelos epidemiológicos o de fallos latentes (Reason, 1990), donde los accidentes se analizan como resultado de fallos activos (inseguros) y condicionales latentes o inseguras, las cuales se caracterizan por ser accidentes que están a expectativa de que ocurran. El objetivo en este modelo es enfocarse en cómo la empresa contribuye a la organización, gestión y construcción de barreras para evitar los accidentes, y considera el error del ser humano como un efecto y no una causa (Instituto Regional de Seguridad y Salud en el Trabajo, 2016). A su vez, entre los modelos más recientes se encuentra el modelo de sistemático avanzado (Dekker, 2005), el cual resalta que los accidentes son consecuencias no esperadas de acciones normales y no por errores del ser humano de manera directa, es decir, puede que el empleado cometa un error, pero la combinación de distintas variables es las que genera el accidente como tal (Instituto Regional de Seguridad y Salud en el Trabajo, 2016).

Desde el punto de vista empírico, las investigaciones tienden a considerar factores desencadenantes de los accidentes relacionados con las condiciones de trabajo y puesto de trabajo (causas micro), con las relaciones laborales (macro) y con las características 
individuales. Gómez (2007) realiza un análisis de la relación salud-trabajo frente a las nuevas realidades y condiciones de trabajo: la globalización e interdependencia entre las empresas, la flexibilización del empleo y la intensificación del trabajo, la innovación tecnológica, el proceso de outsourcing (tercerización), los actuales cambios que se dan a nivel demográfico entre los trabajadores, la incorporación de la mujer al mundo del trabajo remunerado, la desaparición y fusión de empresas, entre otros. Por otro lado, Bolívar, Daponte, López y Mateo (2009), mediante un análisis bivariante y multivariante, buscan estimar la probabilidad de lesión grave, muy grave o mortal asociada con las características del trabajador, características del lugar de trabajo, condiciones de empleo (incluyendo temporalidad y antigüedad) y las condiciones de trabajo. Los resultados muestran que las lesiones más graves se asocian con el sexo masculino, con empresas de menos de 10 empleados y con el hecho de que las labores requieran desplazamientos durante la jornada de trabajo. La antigüedad en el puesto de trabajo no aparece como determinante de la gravedad de las lesiones.

En esta misma línea, García y Montuega (2009), buscan investigar la influencia de algunas características, tanto de los trabajadores como de los puestos de trabajo, en la incidencia de accidentes laborales por categorías (leves, graves, mortales y totales) a partir de un modelo panel con 16 trimestres y con los accidentes de trabajo como unidad de análisis agregados en dos formas: por comunidades autónomas y ocupación, y por ramas de actividad y ocupación. Los resultados arrojan que un mayor porcentaje de trabajadores con contrato indefinido, del sector público, con estudios secundarios o que han recibido cursos de formación se asocian con una menor incidencia de accidentes, mientras que una mayor proporción de varones o extranjeros se relaciona con una mayor incidencia. Una mayor experiencia laboral reduce el riesgo de accidente grave o mortal, mientras que más horas de trabajo lo aumentan.
Rubiales, Agudelo, López y Ronda (2010), a partir de la Encuesta Española de Condiciones de Trabajo, encuentran que, en España, los inmigrantes que proceden de países con bajo Índice de Desarrollo Humano (IDH) tienen mayor riesgo de sufrir accidentes de trabajo que los españoles. No obstante, la prevalencia más elevada se encontró en mujeres, en trabajadores jóvenes, en aquellos con contratos temporales, en el sector privado y en ocupaciones de menor cualificación.

Por otro lado, Pérez y Muñoz (2014), a partir de los reportes de accidentabilidad laboral de una Administradora de Riesgos Laborales, pusieron en evidencia (mediante un análisis univariado y un modelo de regresión logística) que las variables que explicaron con más fuerza el accidente de trabajo mortal fueron: sexo masculino y aquellas con mayor presencia de riesgo, como: accidentarse laboralmente en algún medio de transporte, por violencia, por electricidad, entre otros mecanismos.

Para el caso de estudios que se enfocaron únicamente en los factores de influencia de trabajadores formales, se encuentra que Hoffmeister, Vidal, Vallebuona, Ferrer, Vásquez y Núñez (2014), mediante análisis descriptivo de variables de salud y factores de riesgo, y regresiones logísticas para la ocurrencia de enfermedades y accidentes, encontraron que a mayor edad, ser sedentario y ser hombre presenta mayores probabilidades de ausentarse del trabajo por algún tipo de accidente o enfermedad, variando de acuerdo al tipo de actividad económica en la cual se encontrara la empresa.

Ahora bien, para el caso de Cartagena se trae a colación el estudio de Bedoya (2015), el cual, por medio de un estudio de corte descriptivo y transversal, evalúa una serie de indicadores y hace una revisión de datos discriminados por tipos de accidentes en una empresa metalmecánica, encontrando que, en su mayoría, los más afectados son empleados masculinos vinculados directamente con la empresa con cargos de aprendiz y ayudante. Posteriormente, Bedoya, Severiche, Sierra 
y Osorio (2018), para el caso del sector de la construcción, tuvieron en cuenta un mayor número de variables y obtuvieron resultados como que el promedio de accidentados tiene una edad de 39 años y que aquellas personas en unión libre, que se ubiquen en estrato socioeconómico bajo y que tengan un nivel primario de educación son más propensas a accidentes; por otro lado, se presenta que los técnicos y tecnólogos tienen menor tendencia a accidentarse.

En esta misma línea, González, Bonilla, Quintero, Reyes y Chavarro (2016), en la ciudad de Neiva, Huila, analizan las causas de los accidentes en dos proyectos de construcción con datos de su Administradora de Riesgos Laborales por medio de un modelo de Frank E. Bird. Entre las causas básicas, se observaron: los factores personales como falta de juicio, escasa coordinación, entrenamiento inicial inadecuado, altura, peso, fatiga, entre otras; por otro lado, los factores laborales que se resaltan son: programación insuficiente, entrenamiento deficiente, evaluación errónea de las necesidades y riesgos, entre otros. A su vez, se resalta que la edad con mayor presencia de accidentes laborales es entre los 18 y 35 años, abarcando el 65\% de los casos de accidentes.

A nivel internacional, se encuentra Barrera, González y Pérez (2016) quienes analizan el comportamiento de la accidentabilidad laboral en distintas empresas, centrándose en elementos como: sexo, edad, antigüedad en las empresas, día de la semana de ocurrencia del suceso, así como, los índices de incidencia y frecuencia. A su vez, incluyen variables como la planificación y control, que son poco estudiados en el análisis de los accidentes de trabajo, resaltando que son explicados por variables físicas (del trabajo) y factores organizacionales, como también lo ponen en evidencia Pietilä, Räsänen, Reiman, Ratilainen y Helander (2017).

Los autores Carrasco y Donari (2016), por el método del árbol de causas (Rodríguez de Prada, 2012), buscan comprender, de forma inductiva, los errores que llevaron a que ocu- rriera el accidente y sus consecuencias, logrando prevenir los futuros hechos causantes de los accidentes. Analizan los mismos por 18 ramas de actividad económica, encontrando, para el año 2014, 4.438 accidentes, principalmente en: construcción $(20,2 \%)$, industrias manufactureras $(16,2 \%)$, comercio $(11,6 \%)$, agricultura, ganadería, caza y silvicultura $(9,9 \%)$, entre otros. Se analizaron variables como el sexo, tiempo de antigüedad en la empresa, cargo, entre otras. A su vez, se diferenciaron tres grandes causas: organizacionales, personales y técnicas. Entre las causas organizacionales de los accidentes laborales se encuentran: la realización de tareas (38,7\%), la inexistencia o deficiencia en los procedimientos de trabajo $(24,5 \%)$ y el método de trabajo y la falta de vigilancia $(18,21 \%)$, entre otros. Entre las causas personales se encuentran: factores de comportamiento del trabajador $(77,8 \%)$ y factores intrínsecos de salud o capacidades $(22,2 \%)$; asimismo, la organización del trabajo representa el 35,3\% de las causas de los accidentes. Y por último, entre las causas técnicas de los accidentes laborales se pueden observar principalmente: elementos y dispositivos de protección de máquinas $(24,2 \%)$, diseño, ubicación y mantenimiento de máquinas $(15,2 \%)$, de otros equipos de trabajo $(12,1 \%)$, entre otros.

Un análisis especial ha recibido el estudio de las nuevas relaciones de trabajo sobre la accidentalidad. La indagación muestra que aunque persisten los riesgos laborales clásicos, cada vez es mayor el protagonismo de los factores de riesgo psicosocial y estrés laboral.

\section{Metodología}

Con el objetivo de analizar las causas de los accidentes de trabajo en la ciudad de Cartagena en el periodo 2009 - 2012, el proceso metodológico se enmarca en un enfoque cuantitativo y direccionado al paradigma positivista o empírico. El alcance de la investigación es de tipo correlacional y de acuerdo con el espacio temporal (en un periodo de tiempo de cuatro años) es sincrónica o transversal. 
A su vez, es una investigación no experimental, en la cual, para analizar la relación entre las variables dado del periodo de estudio, se tiene en cuenta un diseño transversal correlacional orientado a la obtención de relaciones entre variables escogidas de acuerdo con las referencias teóricas mencionadas en el apartado anterior, por medio del método científico en la línea de Kerlinger (1981). La información de las variables contempladas en el modelo es tomada de las estadísticas de accidentalidad de Fasecolda e información de la Gran Encuesta Integrada de Hogares (GEIH). Para el análisis de la información de accidentabilidad en Cartagena, se utiliza un modelo panel cuya unidad de análisis son las ramas de actividad, que se describe a continuación.

A partir de Montero (2011), Cameron y Trivedi (2010) y Greene (2002), se tiene que la forma funcional general para la estimación de un modelo panel es (ecuación 1):

$$
\begin{aligned}
& y_{i t}=\alpha_{i t}+x_{i t} B+\varepsilon_{i t} \\
& \mu_{i j}=a_{i t}+\varepsilon_{i t}
\end{aligned}
$$

Donde es la heterogeneidad o el efecto individual, las cuales son tomadas constantes en el tiempo $t$; es el conjunto de regresores; y es el error idiosincrático.

Como referentes metodológicos para esta investigación se tienen, de acuerdo con las consideraciones de los autores para la variable independiente, tres enfoques del modelo: agrupado, de efectos fijos y de efectos variables o aleatorios. En este orden de ideas, se estima una regresión agrupada por el método de mínimos cuadrados ordinarios cuando no hay correlación entre el error y las regresoras $\operatorname{cov}\left(x_{i t}, \mu_{i t}\right)=0 ;$ sin embargo, cuando hay correlación, la regresión agrupada conduce a estimaciones sesgadas. Una de las causas más frecuentes de correlación es el error de especificación por variables omitidas o la existencia de cualidades inobservables del individuo. Este problema puede solucionarse con la estimación de un modelo de efectos fijos o de efectos aleatorios (Montero, 2011; Cameron y Trivedi, 2010; Greene, 2002).
En el modelo de efectos fijos, se supone que las diferencias entre las distintas unidades de análisis no son aleatorias sino constates en el tiempo, por lo cual se estima un intercepto diferente para cada unidad. Asimismo, en este modelo se permite que $a_{i t}$ esté correlacionada con las regresoras, lo que permite una forma limitada de endogeneidad; no obstante, las regresoras no están correlacionadas con el error idiosincrático $\varepsilon_{i t}$. Este modelo implica que $E\left(y_{i t} \mid a_{i t}, x_{i t}\right)=a_{i t}+x_{i t}$ (Montero, 2011; Cameron y Trivedi 2010; Greene, 2002).

En el modelo de afectos aleatorios, se asume que el término $a_{i t}$ es aleatorio, es decir, en lugar de considerar que $a_{i t}$ es fija y contante en el tiempo para cada unidad de análisis, se supone que es una variable aleatoria con media $a_{i t}$ y varianza $\left(a_{i t}\right) \neq 0$ para cada unidad (Montero, 2011; Cameron y Trivedi 2010; Greene, 2002).

Para el análisis de las causas de la accidentalidad en Cartagena, se estiman un modelo agrupado, uno de efectos fijos y otro de efectos aleatorios. Para la selección entre el modelo agrupado y el de efectos aleatorios, se hace uso de la prueba Breusch and Pagan bajo la hipótesis nula de que la varianza del error es cero. Si esta hipótesis se rechaza, se elige el modelo de efectos aleatorios. Asimismo, se realiza una prueba $F$ restrictiva para elegir entre el modelo agrupado y de efectos fijos, bajo la hipótesis nula de que $a_{i t}=0$. Finalmente, la selección entre el modelo de efectos fijos y aleatorios se realiza con la prueba de Hausman con hipótesis nula que $a_{i t}=0$, que, de rechazarse, muestra evidencia a favor del modelo de efectos fijos (Montero, 2011; Cameron y Trivedi 2010; Greene, 2002).

Finalmente, como variables explicativas se encuentran aquellas que se relacionan directamente con las condiciones de trabajo en cada sector económico (causas micro), otras que reflejan la organización de trabajo (causas macro) y aquellas que permiten controlar por características personales de los trabajadores. En síntesis, las variables a incluir en el modelo econométrico se encuentran en la tabla 1. 
TABLA 1

Operacionalización de variables

\begin{tabular}{|c|c|c|}
\hline Variable & Indicador & Fuente \\
\hline \multicolumn{3}{|c|}{ Variable dependiente } \\
\hline Accidentalidad & $\begin{array}{l}\text { Tasa de accidentalidad } \\
\text { Número de accidentes / número de trabajadores *100 }\end{array}$ & FASECOLDA \\
\hline \multicolumn{3}{|c|}{ Variables independientes } \\
\hline Edad & Edad promedio de trabajadores & DANE \\
\hline Género & Porcentaje de hombres & DANE \\
\hline Educación superior & $\begin{array}{l}\text { Porcentaje de trabajadores con educación técnica, } \\
\text { tecnológica, universitaria o de posgrado }\end{array}$ & DANE \\
\hline Contrato indefinido & Porcentaje de trabajadores con contrato indefinido & DANE \\
\hline Trabajadores directos & Porcentaje de trabajadores directos & DANE \\
\hline Antigüedad & Número de meses promedio en la empresa & DANE \\
\hline $\begin{array}{l}\text { Trabajadores de empresas con } \\
\text { menos de } 10 \text { empleados }\end{array}$ & $\begin{array}{l}\text { Porcentaje de trabajadores en empresas de menos de } 10 \\
\text { empleados }\end{array}$ & DANE \\
\hline Trabajadores secundarios & Porcentaje de trabajadores segundarios & DANE \\
\hline Ingresos laborales & Ingreso semanal promedio & DANE \\
\hline
\end{tabular}

Fuente: diseño de los autores

\section{Resultados}

\section{La accidentalidad de Cartagena en cifras}

La magnitud y evolución de la accidentalidad en Cartagena muestra un panorama preocupante. El número de accidentes laborales presentó un aumento del 19,30\% del año 2009 al año 2012, lo que representa un incremento aproximado de un $6 \%$ promedio anual. El número de lesiones fatales se redujo durante este periodo en $3 \%$ anual. Como consecuencia de los accidentes laborales, se reconocen en promedio 13 pensiones de invalidez y se realizan 336 indemnizaciones anuales (Tabla 2).

En términos de índice de accidentalidad, en la ciudad de Cartagena se accidentaron, durante el periodo de análisis, aproximadamente 7.7 trabajadores que se encuentran afiliados en el sistema general de riesgos profesionales, con un punto máximo en el año 2010 que se situó en 8.4 (figura 1). Si bien Cartagena durante los años 2009 y 2011 registró las mayores tasas de accidentalidad de las cinco principales ciudades colombianas, ésta se situó, durante el año 2012, por debajo del promedio nacional y de
TABLA 2

Cartagena: estadísticas de accidentalidad laboral, 2009 - 2012

\begin{tabular}{ccccc}
\hline Accidentalidad & 2009 & 2010 & 2011 & 2012 \\
\cline { 1 - 4 } $\begin{array}{c}\text { Número de } \\
\text { accidentes }\end{array}$ & 12.030 & 13.741 & 14.882 & 14.352 \\
$\begin{array}{c}\text { Número de } \\
\text { muertes }\end{array}$ & 18 & 24 & 6 & 8 \\
$\begin{array}{c}\text { Total pensiones } \\
\text { de invalidez }\end{array}$ & 9 & 12 & 11 & 19 \\
$\begin{array}{c}\text { Total } \\
\text { indemnizaciones } \\
\text { pagadas }\end{array}$ & 229 & 248 & 314 & 553 \\
\cline { 1 - 2 }
\end{tabular}

Fuente: cálculo y diseño de las autoras con base en información de Fasecolda.

ciudades como Cali (7.36), Medellín (7.27) y Barranquilla (6.81).

Por clases de riesgos, se observa una reducción en el índice de accidentalidad durante el año 2012 con respecto al año inmediatamente anterior. Los trabajadores que tradicionalmente presentan mayor tendencia a accidentarse son clasificados, por el Decreto 1295 de 1994, como de riesgo medio, que comprende las actividades que desarrollan procesos como, por ejemplo, manufacturación de automotores; de artículos de cuero; de agujas; fabricación 


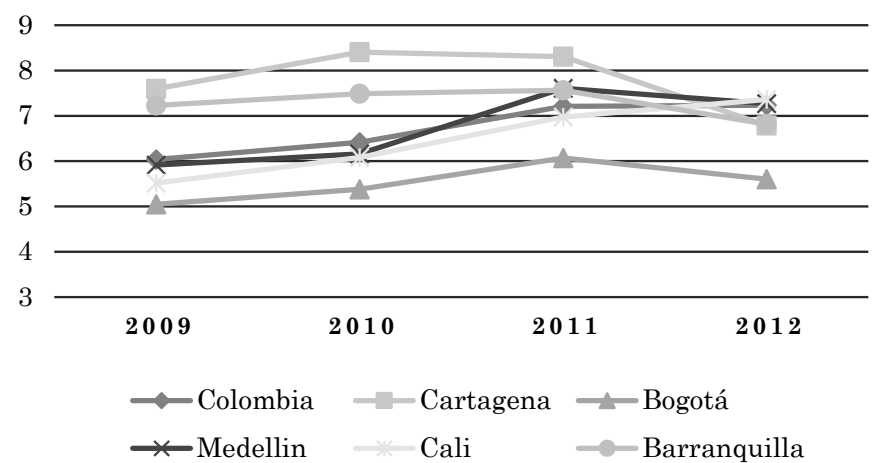

Figura 1. Colombia y cinco principales ciudades: índice de accidentalidad, 2009 - 2012 Fuente: cálculo y diseño de las autoras con base en información de Fasecolda

de alcoholes; alimentos; entre otros. A este grupo, le siguen aquellos que tienen un nivel alto de riesgo, que comprende areneras; manejo de asbesto; bomberos; manejo de explosivos; construcción; explotación petrolera; entre otros. Es de destacar la tendencia decreciente en la accidentalidad entre los trabajadores clasificados en estos dos grupos de riesgos (figura 2).

Por actividad económica, se encuentra que el sector de la industria manufacturera y el de hoteles y restaurantes tradicionalmente presentan mayores índices de accidentalidad, situados en todos los años de análisis por encima de 11 trabajadores accidentados por cada 100 que se encuentran afiliados en el sistema general de riesgos profesionales.

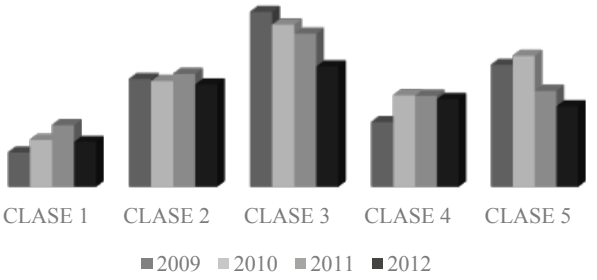

Figura 2. Cartagena: índice de accidentalidad según clase de riesgo, 2009 - 2012

Fuente: cálculo y diseño de las autoras con base en información de Fasecolda

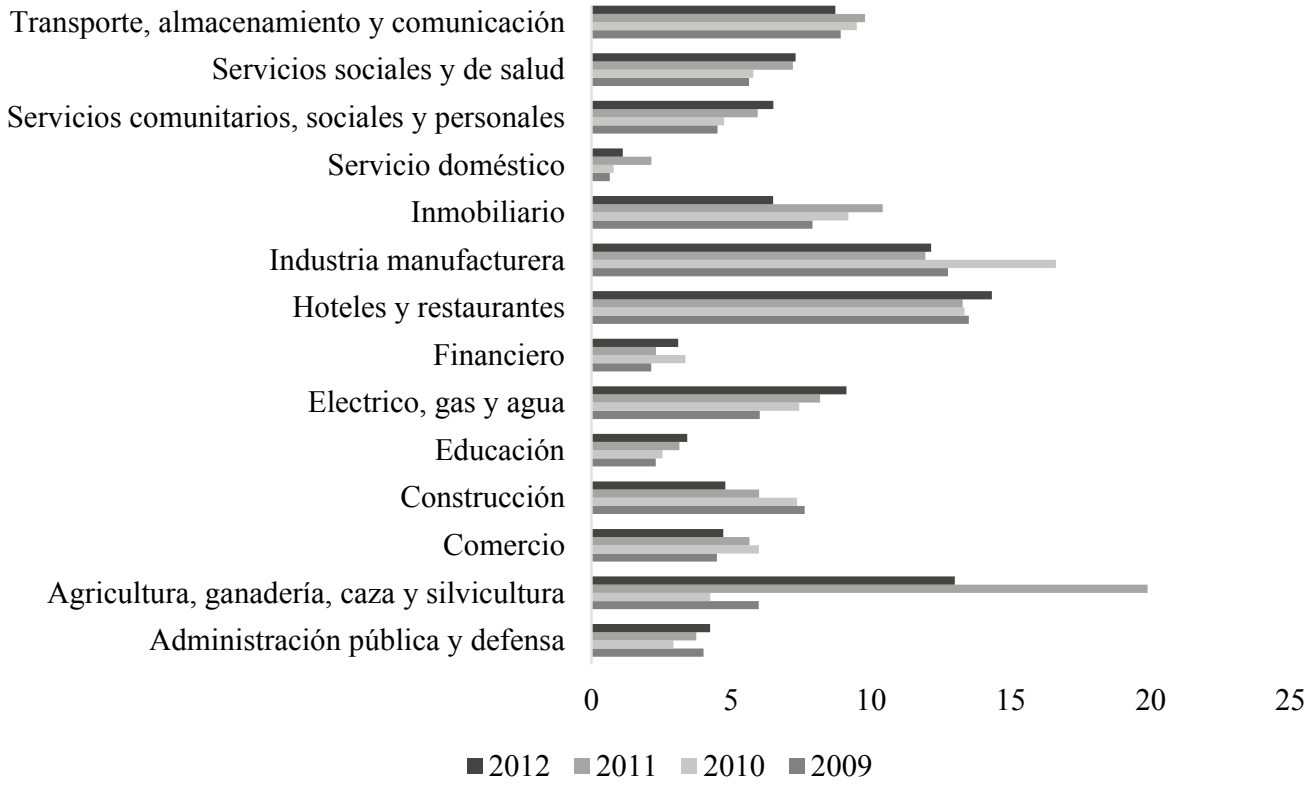

Figura 3. Cartagena: índice de accidentalidad según actividad económica, 2009 - 2012

Fuente: cálculo y diseño de las autoras con base en información de Fasecolda 
En promedio, en la industria manufacturera se reportaron 13.3 accidentes por cada 100 trabajadores y en el sector de hoteles y restaurantes, se reportaron 13.6. Los sectores con menor índice de accidentalidad son el financiero y el servicio doméstico (figura 3).

$\mathrm{Al}$ analizar las variables en series trimestrales (tabla 3), se encuentra que en promedio, por rama de actividad económica, ocurren aproximadamente dos (2) accidentes laborales por cada 100 trabajadores afiliados. Además, la variación del índice de accidentalidad entre las ramas económicas (between) es mayor que la variación en el tiempo en cada una de estas (within), situación predominante en el conjunto de variables independientes consideradas en el modelo.

En la figura 4, se presenta la evolución temporal, desde el primer trimestre de 2009 al cuarto trimestre de 2012, del índice de accidentalidad en cada rama de actividad económica, mostrando que, en general, la industria manufacturera, el sector de hoteles y restaurantes y el sector agropecuario son los que tradicionalmente reportan mayores índices de accidentalidad.

TABla 3

Cartagena: variables por rama de actividades, series trimestrales, 2009 - 2012

\begin{tabular}{|c|c|c|c|c|c|c|}
\hline Variable & & Media & Desv. Estand. & Min & Max & Observaciones \\
\hline \multirow{3}{*}{$\begin{array}{c}\text { Índice de } \\
\text { accidentalidad }\end{array}$} & overall & 1,71 & 1,11 & 0,00 & 6,29 & $\mathrm{~N}=245$ \\
\hline & between & & 0,94 & 0,29 & 3,42 & $\mathrm{n}=16$ \\
\hline & within & & 0,63 & $-0,49$ & 4,97 & $\mathrm{~T}=16$ \\
\hline \multirow[t]{3}{*}{ Edad } & overall & 36,68 & 3,26 & 20,00 & 48,00 & $\mathrm{~N}=245$ \\
\hline & between & & 1,99 & 33,09 & 41,91 & $\mathrm{n}=16$ \\
\hline & within & & 2,60 & 21,55 & 49,55 & $\mathrm{~T}=16$ \\
\hline \multirow{3}{*}{ Sexo (\%) } & overall & 59,63 & 26,27 & 0,00 & 100,00 & $\mathrm{~N}=245$ \\
\hline & between & & 24,73 & 2,65 & 89,98 & $\mathrm{n}=16$ \\
\hline & within & & 10,63 & $-24,75$ & 78,90 & $\mathrm{~T}=16$ \\
\hline \multirow{3}{*}{$\begin{array}{l}\text { Educación } \\
\text { superior (\%) }\end{array}$} & overall & 52,70 & 24,12 & 0,00 & 100,00 & $\mathrm{~N}=245$ \\
\hline & between & & 21,47 & 5,60 & 83,61 & $\mathrm{n}=16$ \\
\hline & within & & 12,01 & $-20,21$ & 114,41 & $\mathrm{~T}=16$ \\
\hline \multirow{3}{*}{$\begin{array}{c}\text { Contrato } \\
\text { indefinido (\%) }\end{array}$} & overall & 59,91 & 22,54 & 0,00 & 100,00 & $\mathrm{~N}=245$ \\
\hline & between & & 19,48 & 1,17 & 90,26 & $\mathrm{n}=16$ \\
\hline & within & & 11,85 & $-9,88$ & 102,32 & $\mathrm{~T}=16$ \\
\hline \multirow{3}{*}{$\begin{array}{l}\text { Trabajadores } \\
\text { directos (\%) }\end{array}$} & overall & 81,31 & 17,19 & 0,00 & 100,00 & $\mathrm{~N}=245$ \\
\hline & between & & 11,23 & 55,18 & 94,79 & $\mathrm{n}=16$ \\
\hline & within & & 13,17 & $-6,19$ & 126,13 & $\mathrm{~T}=16$ \\
\hline
\end{tabular}


Cont...

\begin{tabular}{|c|c|c|c|c|c|c|}
\hline Variable & & Media & Desv. Estand. & Min & Max & Observaciones \\
\hline \multirow{3}{*}{ Antigüedad } & overall & 71,64 & 35,67 & 2,00 & 360,00 & $\mathrm{~N}=245$ \\
\hline & between & & 24,05 & 39,48 & 125,94 & $\mathrm{n}=16$ \\
\hline & within & & 26,70 & $-16,75$ & 341,25 & $\mathrm{~T}=16$ \\
\hline \multirow{3}{*}{$\begin{array}{l}\text { Trabajadores } \\
\text { en empresas } \\
\text { de menos } 10 \\
\text { empleados }(\%)\end{array}$} & overall & 13,47 & 15,27 & 0,00 & 100,00 & $\mathrm{~N}=245$ \\
\hline & between & & 13,66 & 0,00 & 39,56 & $\mathrm{n}=16$ \\
\hline & within & & 7,54 & $-10,25$ & 89,75 & $\mathrm{~T}=16$ \\
\hline \multirow{3}{*}{$\begin{array}{c}\text { Trabajadores } \\
\text { secundarios (\%) }\end{array}$} & overall & 2,21 & 2,96 & 0,00 & 17,36 & $\mathrm{~N}=245$ \\
\hline & between & & 2,25 & 0,00 & 9,17 & $\mathrm{n}=16$ \\
\hline & within & & 2,00 & $-2,31$ & 12,97 & $\mathrm{~T}=16$ \\
\hline \multirow{3}{*}{ Ingresos laborales } & overall & 0,98 & 0,62 & 0,25 & 7,80 & $\mathrm{~N}=240$ \\
\hline & between & & 0,41 & 0,38 & 1,89 & $\mathrm{n}=16$ \\
\hline & within & & 0,48 & 0,03 & 7,15 & $\mathrm{~T}=16$ \\
\hline
\end{tabular}

Fuente: cálculo y diseño de las autoras a partir de información de Fasecolda y Gran Encuesta Integrada de Hogares.

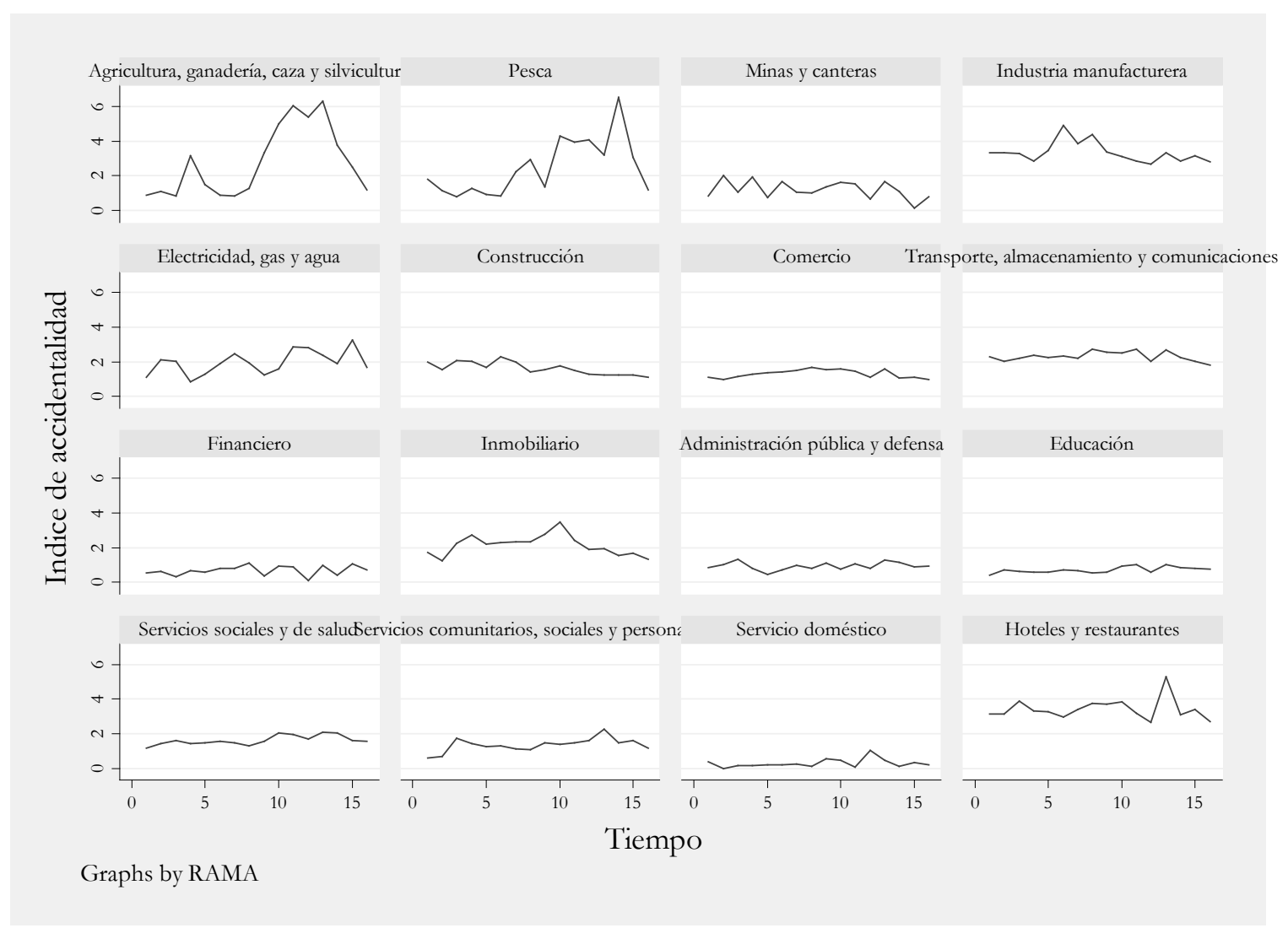

Figura 4. Cartagena: indice de accidentalidad, series trimestrales 2009 - 2012

Fuente: diseño de las autoras a partir de información de Fasecolda y Gran Encuesta Integrada de Hogares 
TABLA 4

Resultados de las estimaciones econométricas

\begin{tabular}{|c|c|c|c|}
\hline $\begin{array}{c}\text { Variables / } \\
\text { Modelo } \\
\end{array}$ & OLS & FE & $\mathrm{RE}$ \\
\hline \multirow[t]{2}{*}{ Edad } & $-0,001$ & 0,004 & 0,001 \\
\hline & 0,023 & 0,023 & 0,022 \\
\hline \multirow[t]{2}{*}{ Sexo } & $0,013^{* *}$ & $0,011^{*}$ & $0,012^{*}$ \\
\hline & 0,005 & 0,005 & 0,005 \\
\hline \multirow[t]{2}{*}{$\begin{array}{l}\text { Educación } \\
\text { superior }\end{array}$} & $-0,017^{* * *}$ & $-0,018^{* * * *}$ & $-0,017^{* * *}$ \\
\hline & 0,005 & 0,005 & 0,005 \\
\hline \multirow[t]{2}{*}{$\begin{array}{c}\text { Contrato } \\
\text { indefinido }\end{array}$} & $-0,011^{*}$ & $-0,011^{*}$ & $-0,011^{*}$ \\
\hline & 0,005 & 0,005 & 0,005 \\
\hline \multirow[t]{2}{*}{$\begin{array}{c}\text { Trabajadores } \\
\text { directos }\end{array}$} & $-0,002$ & $-0,002$ & $-0,002$ \\
\hline & 0,004 & 0,004 & 0,004 \\
\hline \multirow[t]{2}{*}{ Antigüedad } & $-0,010^{* *}$ & $-0,010^{*}$ & $-0,010^{* *}$ \\
\hline & 0,004 & 0,004 & 0,004 \\
\hline \multirow[t]{2}{*}{$\begin{array}{c}\text { Trabajadores } \\
\text { en empresas de } \\
10 \text { empleados o } \\
\text { menos }\end{array}$} & 0,003 & $-0,001$ & 0,002 \\
\hline & 0,007 & 0,007 & 0,007 \\
\hline \multirow[t]{2}{*}{$\begin{array}{l}\text { Trabajadores } \\
\text { secundarios }\end{array}$} & $-0,011$ & $-0,007$ & $-0,009$ \\
\hline & 0,022 & 0,022 & 0,022 \\
\hline \multirow[t]{2}{*}{$\begin{array}{l}\text { Ingresos } \\
\text { laborales }\end{array}$} & $-0,055$ & $-0,05$ & $-0,053$ \\
\hline & 0,09 & 0,089 & 0,089 \\
\hline \multirow[t]{2}{*}{ Constante } & $1,766^{*}$ & $1,647^{*}$ & $1,715^{*}$ \\
\hline & 0,918 & 0,918 & 0,931 \\
\hline $\mathrm{N}$ & 224 & 224 & 224 \\
\hline $\mathrm{r} 2$ & & 0,131 & \\
\hline r2_o & & 0,165 & 0,220 \\
\hline r2_b & & 0,133 & 0,202 \\
\hline $\mathrm{r} 2$ _w & & 0,131 & 0,130 \\
\hline sigma_u & & 0,928 & 1,054 \\
\hline sigma_e & & 0,594 & 0,594 \\
\hline rho & & 0,709 & 0,759 \\
\hline \multicolumn{4}{|c|}{${ }^{*} \mathrm{p}<0,05,{ }^{* *} \mathrm{p}<0,01,{ }^{* * *} \mathrm{p}<0,001$} \\
\hline
\end{tabular}

Fuente: cálculo y diseño de las autoras a partir de información de Fasecolda y Gran Encuesta Integrada de Hogares.

\section{Resultados econométricos}

Para indagar sobre los factores determinantes de la accidentalidad laboral en Cartagena, se inicia con la estimación de un modelo agrupado por el método de mínimos cuadrados ordinarios para todas las ramas de actividad económica y trimestres entre 2009 y 2012 , así como un modelo de efectos fijos y de efectos aleatorios para, posteriormente, hacer la selección del mejor modelo a partir de las relaciones transversales y temporales de las variables. En la tabla 4, se presentan los resultados de estas estimaciones econométricas.

Con el objetivo de seleccionar entre el modelo de efectos fijos y la regresión agrupada, se examina el $F$ restringido que se obtiene del primero de los modelos. Estos resultados se muestran en la tabla 5 .

TABLA 5

\section{Prueba $\boldsymbol{F}$ restringida}

\begin{tabular}{ccc}
\hline $\begin{array}{c}\mathrm{F} \\
\text { restringido }\end{array}$ & $\mathrm{F}$ & P-Value \\
\hline all $\mathrm{u}_{\mathrm{i}}=0$ & $\mathrm{~F}(15,233)=24,18$ & 0,0000 \\
\hline
\end{tabular}

Fuente: cálculo y diseño de las autoras a partir de información de Fasecolda y Gran Encuesta Integrada de Hogares.

El p-value de $F$ indica que se puede rechazar la hipótesis nula de que la heterogeneidad o efecto individual es cero $\left(a_{i}=0\right)$, por lo que es preferible usar el método de efectos fijos al modelo agrupado.

La tabla 6, muestra los resultados de la prueba de Breusch y Pagan la cual permite seleccionar entre el modelo agrupado y la regresión de efectos aleatorios. Dado que la significatividad del estadístico chicuadrado rechaza la hipótesis nula de que la varianza del error es cero, es preferible el modelo de efectos aleatorios. 
TABla 6

Prueba de multiplicador Lagrangian de Breusch y Pagan para efectos aleatorios

\begin{tabular}{ccc}
\hline \multicolumn{2}{l}{ IACC $[$ RAMA,t] $=\mathrm{Xb}+\mathrm{u}[\mathrm{RAMA}]+\mathrm{e}[\mathrm{RAMA}, \mathrm{t}]$} \\
\hline & Var & $\mathrm{sd}=\mathrm{sqrt}($ Var $)$ \\
\hline $\mathrm{IACC}$ & 1,30 & 1,14 \\
$\mathrm{e}$ & 0,35 & 0,59 \\
$\mathrm{u}$ & 1,11 & 1,05 \\
\hline
\end{tabular}

Test: $\operatorname{Var}(\mathrm{u})=0$

$\operatorname{chi} 2(01)=445.15$

Prob $>$ chi2 $=0.0000$

Fuente: Cálculo y diseño de las autoras a partir de información de Fasecolda y Gran Encuesta Integrada de Hogares.

Por otra parte, la selección entre el modelo de efectos fijos y el de efectos aleatorios se realiza a partir de la prueba de Hausman, cuyos resultados se observan en la tabla 7. La prueba de Hausman no permite rechazar la hipótesis nula de que las diferencias en los coeficientes son no sistemáticas, por lo cual es preferible el modelo de efectos aleatorios.

Finalmente, en la tabla 8 se presentan los resultados de los determinantes de la accidentalidad laboral en la ciudad de Cartagena a partir de un modelo panel de efectos aleatorios durante el periodo 2009 - 2012. La selección de un modelo de efectos aleatorios en lugar de la regresión agrupada implica que hay una correlación entre las características inobservables de cada grupo de trabajadores y el conjunto de regresores, lo que permite comprobar de una manera indirecta la importancia de las características propias del trabajo en la ocurrencia de la accidentalidad. Asimismo, la selección del modelo de efectos aleatorios al de efectos fijos implica que hay que considerar la heterogeneidad individual en el tiempo.

TABla 7

Prueba de Hausman para efectos fijos

\begin{tabular}{ccccc}
\hline Variables & \multicolumn{5}{c}{ Coeficientes } \\
\hline & $(\mathrm{b})$ & $(\mathrm{B})$ & $(\mathrm{b}-\mathrm{B})$ & sqrt(diag(V_b-V_B) \\
& $\mathrm{FE}$ & $\mathrm{RE}$ & Diferencia & S.E. \\
\hline Edad & 0,004 & 0,001 & 0,003 & 0,004 \\
\hline Sexo & 0,011 & 0,012 & $-0,001$ & 0,002 \\
\hline Educación superior & $-0,018$ & $-0,017$ & $-0,001$ & 0,001 \\
\hline Contrato indefinido & $-0,011$ & $-0,011$ & 0,000 & 0,001 \\
\hline Trabajadores directos & $-0,002$ & $-0,002$ & 0,000 & 0,001 \\
\hline Antigüedad & $-0,010$ & $-0,010$ & 0,000 & 0,001 \\
\hline Empresas menos 10 & $-0,001$ & 0,002 & $-0,003$ & 0,003 \\
\hline Trabajadores secundarios & $-0,007$ & $-0,009$ & 0,003 & 0,005 \\
\hline Ingresos laborales & $-0,050$ & $-0,053$ & 0,003 & 0,013 \\
\hline
\end{tabular}

Test: Ho: diferencia en los coeficientes no sistemática

$\operatorname{chi} 2(9)$

$$
=(b-B)^{\prime}\left[\left(V_{-} b-V \_B\right)^{\wedge}(-1)\right](b-B)
$$$$
=3,15
$$

Prob > chi2

$$
=0,9580
$$

Fuente: cálculo y diseño de las autoras a partir de información de Fasecolda y Gran Encuesta Integrada de Hogares. 
TABLA 8

Resultados de la estimación de un modelo de efectos aleatorios

\begin{tabular}{|c|c|c|c|c|c|c|}
\hline \multicolumn{4}{|c|}{ Random-effects GLS regression } & Number of obs & $=$ & 224 \\
\hline \multicolumn{4}{|c|}{ Group variable: RAMA } & Number of groups & $=$ & 15 \\
\hline R-sq: within & \multicolumn{3}{|c|}{$=0,1297$} & Obs per group: min & $=$ & 5 \\
\hline between & \multicolumn{3}{|c|}{$=0,2022$} & avg & $=$ & 14,9 \\
\hline overall & \multicolumn{3}{|c|}{$=0,2201$} & $\max$ & $=$ & 16 \\
\hline & & & & Wald chi2(9) & $=$ & 32,87 \\
\hline \multicolumn{4}{|c|}{$\operatorname{corr}\left(\mathrm{u}_{\mathrm{i}}, \mathrm{X}\right)=0$ (assumed) } & Prob > chi2 & $=$ & 0,0001 \\
\hline IACC & Coef & Std. Err. & $\mathrm{z}$ & $\mathrm{P}>\mathrm{z}$ & {$[95 \%$ Conf. } & Interval] \\
\hline Edad & 0,001 & 0,022 & 0,050 & 0,962 & $-0,043$ & 0,045 \\
\hline Sexo & 0,012 & 0,005 & 2,500 & 0,012 & 0,003 & 0,021 \\
\hline Educación superior & $-0,017$ & 0,005 & $-3,720$ & 0,000 & $-0,027$ & $-0,008$ \\
\hline Contrato indefinido & $-0,011$ & 0,005 & $-2,340$ & 0,019 & $-0,020$ & $-0,002$ \\
\hline Trabajadores directos & $-0,002$ & 0,004 & $-0,510$ & 0,609 & $-0,010$ & 0,006 \\
\hline Antigüedad & $-0,010$ & 0,004 & $-2,700$ & 0,007 & $-0,017$ & $-0,003$ \\
\hline Empresas menos 10 & 0,002 & 0,007 & 0,240 & 0,810 & $-0,012$ & 0,015 \\
\hline $\begin{array}{l}\text { Trabajadores } \\
\text { secundarios }\end{array}$ & $-0,009$ & 0,022 & $-0,430$ & 0,669 & $-0,052$ & 0,033 \\
\hline Ingresos laborales & $-0,053$ & 0,089 & $-0,600$ & 0,550 & $-0,226$ & 0,121 \\
\hline Constante & 1,715 & 0,931 & 1,840 & 0,055 & $-0,110$ & 3,539 \\
\hline sigma_u & 1,054 & & & & & \\
\hline sigma_e & 0,594 & & & & & \\
\hline rho & 0,759 & (frac & on of va & ance due to $\mathrm{u} \_$i) & & \\
\hline
\end{tabular}

Fuente: cálculo y diseño de las autoras a partir de información de Fasecolda y Gran Encuesta Integrada de Hogares.

De acuerdo con los resultados obtenidos, las variables más importantes para explicar la accidentalidad en Cartagena son la proporción de empleados de género masculino, la proporción de empleados con contrato indefinido, la antigüedad en la empresa y la proporción de empleados con educación universitaria. En particular, se encontró que la antigüedad en la empresa y la tenencia de contratos indefinidos como proxys de la estabilidad en el trabajo son uno de los determinantes más robustos en la accidentalidad. Esto permite fortalecer la corriente empírica que le da mayor preponderancia a las nuevas formas de organización en el trabajo sobre la ocurrencia de accidentes.
Es menester mencionar que los resultados son consistentes con la teoría y evidencia empírica sobre los determinantes de la accidentalidad, es decir, las actividades con predominio del género masculino, tienen mayor índice de accidentalidad; asimismo, las actividades en las cuales hay mayor porcentaje de empleados con contrato indefinido, con mayor antigüedad y con educación superior tienen un menor índice. El respaldo de las variables (que se manejan en el modelo presentado en esta investigación) con la literatura se puede evidenciar en algunos estudios, como en: Benavides, Delclos, Benach y Serra (2006) en donde la incidencia de trabajadores a término indefinido es menor a los temporales; Bolívar, 
Daponte, López y Mateo (2009) y Pérez y Muñoz (2014), donde los accidentes de sexo masculino y con empresas de menos de diez trabajadores presentan más accidentes; García y Noruega (2009) y Bedoya, Severiche, Sierra y Osorio (2018) que tienen en cuenta la variable de nivel educativo, sexo y tipo de contrato; Rubiales, Agudelo, López y Ronda (2010) y Barrera, González y Pérez (2016) toman alguna de estas variables como referencia (edad, sexo y tipo de contrato, entre otros).

Cabe resaltar que algunas variables que no se consideraron en este modelo y que se encontraron en la revisión literaria fueron: tipología de la empresa (pública o privada); si requiere desplazamientos en el lugar de trabajo; tipo de ocupación de acuerdo con su cualificación, entre otras.

A su vez, de acuerdo con el índice de accidentabilidad encontrado en la ciudad de Cartagena, se pone en evidencia un contraste positivo con la teoría de la probabilidad sesgada dado que los trabajadores que sufran un accidente presentan mayor o menor probabilidad de ocurrencia, es por ello el comportamiento similar en el periodo de estudio de acuerdo con la clase de riesgo del trabajador, sea en el sector industrial; hoteles y restaurantes; y agricultura, ganadería, caza y silvicultura. Asimismo, se contrasta con el modelo de causalidad derivada de las teorías del modelo de Heinrich (1931) dadas las distintas variables estadísticamente significativas presentes en el modelo estudiado.

\section{Conclusiones}

En esta investigación se realizó un análisis de las causas de los accidentes de trabajo mediante la estimación de un modelo panel cuya unidad de análisis fueron los grupos de trabajadores en la ciudad de Cartagena, Colombia definido por la rama de actividad económica durante el periodo 2009 - 2012; ciudad que tradicionalmen- te presentó mayores índices de empleados accidentados, pero que durante el 2012 redujo este indicador por debajo de la media nacional. Esto, sumado a que Cartagena es una de las ciudades con mayor dinámica económica y de empleo en el país, hace de esta ciudad un interesante caso para analizar la dinámica de la accidentalidad.

A partir de estadísticas descriptivas, se tiene que en Cartagena el número de accidentes laborales se incrementó de 12.030 en el año 2009 a 14.352 en el año 2012, lo que representa un incremento aproximado de un $6 \%$ promedio anual. El número de lesiones fatales se redujo durante este periodo en un 3\% anual. Como consecuencia de los accidentes laborales, se reconocen en promedio 13 pensiones de invalidez y se realizan 336 indemnizaciones anuales.

A nivel econométrico, se desarrollaron esquemas de modelación para el análisis de los factores determinantes de la accidentalidad laboral en Cartagena. Para tal fin, se inició con un modelo agrupado por el método de mínimos cuadrados ordinarios para todas las ramas de actividad económica y trimestres entre 2009 y 2012 , así como un modelo de efectos fijos y de efectos aleatorios para posteriormente hacer la selección del mejor modelo a partir de las relaciones transversales y temporales de las variables. Luego de realizar estas estimaciones, se obtuvo que las variables que más inciden en la accidentalidad laboral en Cartagena son: el género masculino, la tipología de contrato indefinido, la antigüedad en la empresa y la educación universitaria. En particular, se encontró que la antigüedad en la empresa y la tenencia de contratos indefinidos, como elementos proxy de la estabilidad en el trabajo, son uno de los determinantes más robustos en la accidentalidad. Esto permite fortalecer la corriente empírica que le da mayor preponderancia a las nuevas formas de organización en el trabajo sobre la ocurrencia de accidentes. 
Ahora bien, dadas las particularidades en la evolución de la accidentalidad laboral en la ciudad de Cartagena, y luego de evaluar diferentes especificaciones, se estimó un modelo tipo panel con efectos aleatorios. Este modelo permite tomar en cuenta la correlación entre las características inobservables de cada grupo de trabajadores con las variables independientes consideradas, apoyándose así la hipótesis de que la accidentalidad se ve influenciada por las características propias del trabajo.

Es necesario anotar que si bien las características propias del trabajo (factores micro) explican la accidentalidad en la ciudad, también son importantes las nuevas relaciones de trabajo en la ocurrencia de ésta, en particular, de la estabilidad laboral. En esta investigación se presentó evidencia empírica significativa de que la antigüedad en la empresa y tener contrato indefinido tienen una relación inversa con la accidentalidad, lo que se puede atribuir al hecho de que la reducción de errores en la realización de una labor, y, por tanto, de accidentarse, sólo es posible cuando el empleado puede acumular experiencia en una determinada tarea. Esto permite llamar la atención sobre otro de los costos "ocultos" para la sociedad de la flexibilidad en el mercado de trabajo.

Por otro lado, es menester mencionar que el estudio de las causas de la accidentalidad laboral ha sido objeto de discusiones diversas, a partir de las cuales se han derivado formulaciones de teorías y modelos que parten de elementos micro (propios de las condiciones del trabajo) y macro (resultado de las denominadas relaciones de trabajo). No obstante, pese a estos avances entre las limitaciones del estudio se encuentra que en el país es aún un tema en construcción porque no se cuenta con microdatos consistentes para realizar modelaciones robustas, pues las administradoras de riesgos profesionales sólo contienen información sobre la población con cobertura, lo cual es sensible a los cambios en el mercado de trabajo. Dadas estas circunstancias es imposible contar, tanto en Colombia como en el a ciudad de Cartagena, con un perfil epidemiológico de la población cubierta y la distribución de los afiliados por exposición a factores de riesgo.

Por último, entre las contribuciones del estudio a la comunidad académica se encuentra que sirve como referencia literaria para futuras investigaciones que estudien los accidentes laborales en la ciudad de Cartagena o Colombia dada la escasa evidencia literaria encontrada, ya sea para los grupos de investigación de seguridad laboral, mercado laboral, salud ocupacional, entre otras líneas, y también, para las empresas y aseguradoras.

\section{Referencias}

Barrera, A., González, A. y Pérez, D. (2016). Identificación de factores incidentes en la accidentalidad laboral en empresas de Cienfuegos. Ingeniería Industrial, 37(2), 127-137.

Bedoya, E. A. (2015). Comportamiento de la accidentalidad en una empresa metalmecánica en Cartagena, Colombia. NOVA, 13(24), 93-99.

Bedoya, E., Severiche, C. A., Sierra, D. D. y Osorio, I. C. (2018). Accidentalidad laboral en el sector de la construcción: el caso del Distrito de Cartagena de Indias (Colombia), Periodo 2014-2016. Información Tecnológica, 29(1), 193-200.

Benavides, F., Delclos, J., Benach, J. y Serra, C. (2006). Lesiones por accidentes de trabajo, una prioridad en salud pública. Revista Española de Salud Pública, 80(5), 553-565.

Bolívar, J., Daponte, A., López, L. y Mateo, I. (2009). Influencia de las características individuales y de las condiciones laborales en la gravedad de las lesiones por accidentes de trabajo registradas en Andalucía en 2003. Revista Española de Salud Pública, 83, 847-861. 
Botta, N. (2010). Teorías y modelización de los accidentes (3ra ed.). Rosario, Argentina: Red Proteger.

Comunidad Andina. (2003). Decisión 584: Sustitución de la Decisión 547, Instrumento Andino de Seguridad y Salud en el Trabajo. Guayaquil, Ecuador: Sistema de Información sobre Comercio Exterior, Organización de los Estados Americanos. Recuperado de: http:// www.sice.oas.org/trade/junac/decisiones/DEC584s.asp

Cameron, A. C. y Trivedi, P. K. (2010). Microeconometrics Using Stata. College Station, Texas: Stata Press.

Carrasco, C. y Donari, J. (2016). Accidentabilidad laboral: análisis de las causas más recurrentes y factores intervinientes. Santiago de Chile, Chile: Dirección del Trabajo.

Cortez, W. (2003). Accidentes de trabajo y eficacia de los programas preventivos de seguridad e higiene. Revista de Comercio Exterior, 53, 712-722.

Departamento Nacional de Planeación. (1936). Misión, Visión y Origen. Bogotá, Colombia: Departamento Nacional de Planeación (DNP). Recuperado de: https://www.dnp.gov.co/DNP/acercade-la-entidad/Paginas/quienes-somos. aspx

Dekker, S. (2005). Ten questions about human error: A new view of human factors and system safety. Nueva Jersey, Estados Unidos: Lawrence Erlbaum Associates.

Federación de Aseguradoras Colombianas. (2018). Estadísticas del ramo. Bogotá, Colombia: Federeación de Aseguradoras Colombianas (Fasecolda). Recuperado de http:// www.fasecolda.com/index.php/ramos/ riesgos-laborales/estadisticas-del-ramo/
Gallego, M. I. y Correa, J. C. (2000). Indicadores de accidentalidad laboral, normatividad y recomendaciones en Colombia. Revista de la Facultad Nacional de Salud Pública, 18(1), 81-93.

García, I. y Montuega, V. (2009). Causa de los accidentes de trabajo en España: análisis longitunidal con datos de panel. Gaceta Sanitaria, 23(3), 174 - 178.

Gomero, R., LLapyesan, C., Calizaya, L. y Silvera, R. (2005). Accidentes fuera del trabajo: Análisis en el campamento minero de Toquepala 2002-2003. Revista Médica Herediana, 16(2). 107113.

Gómez, I. (2007). Salud laboral: una revisión a la luz de las nuevas condiciones del trabajo. Universitas Psychologica, 6(1), 105-113.

González, A., Bonilla, J., Quintero, M. Reyes, C. y Chavarro, A. (2016). Análisis de las causas y consecuencias de los accidentes laborales ocurridos en dos proyectos de construcción. Revista Ingeniería de Construcción, 31(1), 5-16.

Greene, W. (2002). Econometric Analysis (5th ed.). Upper Saddle River, New Jersey: Prentice Hall.

Heinrich, H. (1931). Industrial Accident Prevention. Nueva York, Estados Unidos: McGraw-Hill.

Hoffmeister, L., Vidal, C., Vallebuona, C., Ferrer, N., Vásquez, P. y Núñez, G. (2014). Factores asociados a accidentes, enfermedades y ausentismo laboral: análisis de una cohorte de trabajadores formales en Chile. Ciencia \& Trabajo, 16(49), 21-27.

Instituto Regional de Seguridad y Salud en el Trabajo. (2016). Metodología y técnicas analíticas para la investigación de accidentes de trabajo. Madrid, España: Fundación Agustín de Betancourt. 
Kerlinger, F. (1981). Enfoque conceptual de la investigación del comportamiento. Ciudad de México, México: Interamericana.

Meliá, J. (2004). El modelo causal psicosocial de los accidentes laborales de la Universidad de Valencia: perspectivas $y$ nuevos retos. Valencia, España: Universidad de Valencia.

Montero, R. (2011). Efectos fijos o aleatorios: test de especificación. Universidad de Granada. Granada, España: Documentos de Trabajo en Economía Aplicada.

Organización Internacional del Trabajo OIT. (2007). Informe para el día mundial de la seguridad y salud en el trabajo. Ginebra, Francia: Oficina Internacional del trabajo, CH-1211 Ginebra 22.

Organización Internacional del Trabajo. OIT. (2012). La Organización Internacional del Trabajo en acción: resultados de desarrollo 2010-2011. Ginebra, Francia: Oficina Internacional del Trabajo.

Pérez, O., M. M. y Muñoz, D., A. M. (2014). Accidentalidad laboral reportada por las empresas afiliadas a una Administradora de Riesgos Laborales, enero - diciembre, 2011. Rev. Fac. Nac. Salud Pública, $32(2), 67-75$.

Pietilä, J., Räsänen, T., Reiman, A., Ratilainen, H. y Helander, E. (2017). Characteristics and determinants of recurrent occupational accidents. Safety Science, 108(1). 269-277. https://doi.org/10.1016/j. ssci.2017.12.020

Raouf, A. (1998). Teoría de las causas de los accidentes. En: Organización Internacional del Trabajo, Enciclopedia de salud y seguridad en el trabajo (56.6). Ginebra, Francia: Organización Internacional del Trabajo.

Reason, J. (1990). Human error. New York, Estados Unidos: Cambridge University Press.

Rodríguez de Prada, A. (2012). Investigación de accidentes por el método del Árbol de Causas ( $2^{\circ}$ ed.). Madrid, España: Instituto Nacional de Seguridad e Higiene en el Trabajo (Gobierno de España).

Rubiales, E., Agudelo, A. A., López, M. J. y Ronda, E. (2010). Diferencias en los accidentes laborales en España según país de procedencia del trabajador. Salud Pública de México, 52(3), 199-206.

Saari, J. (1998). Prevención de accidentes. En: Organización Internacional del Trabajo, Enciclopedia de salud y seguridad en el trabajo (pág. 56). Ginebra, Francia: Organización Internacional del Trabajo.

Wilde, G. (1998). Modelos de accidentes: homeostasis del riesgo. En: Organización Internacional del Trabajo, Enciclopedia de salud y seguridad en el trabajo (p. 3). Ginebra, Francia: Organización Internacional del Trabajo.

Martha Yánez Contreras. Docente titular del programa de Economía de la Universidad de Cartagena e investigadora del Grupo de Investigación de Mercado Laboral de la Facultad de Ciencias Económicas de la Universidad de Cartagena. Magíster en Economía y Magíster en Economía del Medio Ambiente y de los Recursos Naturales de la Universidad de los Andes. Economista de la Universidad de Cartagena (Colombia)

Karina Acevedo González. Magíster en Economía de la Universidad de los Andes. Economista de la Universidad de Cartagena (Colombia). 